Revue internationale P.M.E.

Économie et gestion de la petite et moyenne entreprise

Revue

internationale

PME

\title{
Quatre paradigmes sur le district industriel
}

\section{Vincenzo Vagaggini et Elea Olivetti}

Volume 2, numéro 2-3, 1989

URI : https://id.erudit.org/iderudit/1007938ar

DOI : https://doi.org/10.7202/1007938ar

Aller au sommaire du numéro

Éditeur(s)

Presses de l’Université du Québec

ISSN

0776-5436 (imprimé)

1918-9699 (numérique)

Découvrir la revue

Citer cet article

Vagaggini, V. \& Olivetti, E. (1989). Quatre paradigmes sur le district industriel. Revue internationale P.M.E., 2(2-3), 253-272. https://doi.org/10.7202/1007938ar

\section{Résumé de l'article}

Après sa redécouverte, on a attribué au "district industriel» une "paternité» dans les travaux d'Alfred Marshall sur les économies externes. Plus récemment, à l'aide de bases théoriques plus solides, on a eu recours à la théorie transactionnelle des rapports entre les entreprises. La tentative de récupérer la totalité des relations a conduit au recours à la théorie de la complexité. Aucune de ces trois théories ne permet de comprendre en profondeur la dimension culturelle que tous considèrent comme fondamentale dans le district, mais qui reste en dehors de ces analyses. Nous proposons plutôt de prendre pour base la théorie de l'agir communicatif pour pénétrer en profondeur dans les transformations socio-culturelles du district. 


\title{
Quatre paradigmes sur le district industriel
}

\author{
Vincenzo VAGAGGINI \\ Elea OLIVETTI
}

\begin{abstract}
RÉSUME
Après sa redécouverte, on a attribué au «district industriel" une «paternité" dans les travaux d'Alfred Marshall sur les économies externes. Plus récemment, àl'aide de bases théoriques plus solides, on a eu recours à la théorie transactionnelle des rapports entre les entreprises. La tentative de récupérer la totalité des relations a conduit au recours à la théorie de la complexité. Aucune de ces trois théories ne permet de comprendre en profondeur la dimension culturelle que tous considèrent comme fondamentale dans le district, mais qui reste en dehors de ces analyses. Nous proposons plutôt de prendre pour base la théorie de l'agir communicatif pour pénétrer en profondeur dans les transformations socio-culturelles du district.
\end{abstract}

\section{ABSTRACT}

Following it rediscovery, the aindustrial districtw has been considered responsible for Alfred Marshall's works on the external economies. More recently, in the search for stronger theoretical grounds the transactional theory of relationships among different compagnies has been taken into account. The attempt at recovering the olistic dimension of the relationships has led to the theory of complexity. None of these three paradigms allows to fully comprehend the cultural background, which is widely considered as foundamental factor but which nevertheless is kept excluded from the analysis. The advice emerging from the work is that of supporting the theory of communicative action in order to go deep into the social and cultural changes of the district.

- Vincenzo Vagaggini est responsable des recherches sur le thème «Ambiente, Strategie e mercato» du Centro Formazione/Consulenza Manageriale e Linguistica, de la firme Elea/ Olivetti Information Services. Adresse: Via Pavone, 1 - Banchette (Torino). 


\section{RESUMEN}

Después de su descubrimiento se ha atribuido al distrito industrial una paternidad en los trabajos de Alfred Marshall sobre las economias externas. Muy recientemente, con la ayuda de bases teorricas mas solidas se ha hecho recurso a la teoria transaccional de las relaciones entre las empresas. La tentativa de recuperar la totalidad de las relaciones ha conducido al recurso de la teoria de la complejidad. Ninguna de estas tres teorias permite de comprender con profundidad la dimensión cultural que todos consideran como fundamental en el distrito, pero que queda excluida de estos análisis. Proponemos mas que todo, por base, la teoria del actuar comunicativo para penetrar con produndidad dentro de las transformaciones socioculturales del distrito. 


\section{Introduction}

Après quinze ans de débat sur les districts industriels, il est maintenant possible de construire une histoire tant théorique qu'empirique de ses raffinements successifs et d'en tirer une explication générale suite à l'étonnante dynamique du «modèle italien». Les «quatre paradigmes sur le district industriel» constituent une première approche de cette explication ayant pour base une succession de propositions épistémologiques susceptibles de pénétrer à l'intérieur de ce phénomène même. Ces propositions ne sont pas incompatibles entre elles; elles représentent plutôt une tentative de définir, à l'aide d'instruments théoriques toujours plus raffinés, la totalité complexe qu'est le district industriel. Le district se présente comme un tout économique et social, établi historiquement dans un espace territorial déterminé et donc, dans des frontières relativement claires et définies. Mais c'est justement pour cela qu'il ne se prête pas à des méthodes réductrices objectives en termes nomologiques, à travers la théorisation de ses implications spatiales singulières.

Pour analyser le district, se sont retrouvées dès le début dans le même champ et sous forme de paradigmes fondamentaux toutes les théories économiques essayant l'une après l'autre de représenter le fonctionnement d'un système économique complexe, soit par l'étude des équilibres économiques produits par les comportements micro-économiques rationnels dans des conditions idéales et abstraites (la théorie néo-classique), soit en remontant à la nature, aux causes et aux conséquences du développement économique (la théorie classique) ou encore, en partant des conditions macro-économiques assurant la pleine utilisation des ressources (la théorie keynésienne). Ces théories controversées ont plutôt occulté le phénomène émergeant des systèmes de petites entreprises en tant que moteur du développement industriel italien des années 70, alors que la grande entreprise était entrée en crise et que la gouverne de l'économie italienne devenait de plus en plus problématique.

Dans cet article, mon argumentation suivra le cheminement suivant : premièrement, je partirai de la «découverte» du phénomène du «district industriel» et des premières tentatives pour retrouver, dans l'histoire des théories, un cadre capable de l'expliquer; deuxièmement, j'examinerai une première proposition en vue de pénétrer à l'intérieur des relations mêmes qui structurent le district; troisièmement, j'aborderai le problème de la complexité du district vu ici comme système, en me référant, dans ce cas, à certains auteurs et en essayant d'approfondir leur analyse; quatrièmement, je ferai ressortir les insuffisances des approches antérieures et j'avancerai une explication fondée sur la théorie de la compétence communicative, laquelle incorpore une dynamique sociale et culturelle qui, malgré tous les efforts contraires, est trop souvent vue dans d'autres paradigmes comme extérieure au système des entreprises. 


\section{Le paradigme marshallien des économies externes}

L'analyse du développement industriel italien à base des économies territoriales extrêmement dynamique et fondée sur l'interdépendance de milliers de petites entreprises étroitement intégrées et fonctionnant à l'intérieur d'un contexte social et culturel local, a amené plusieurs économistes à se tourner vers les théories classiques afin d'y trouver un cadre théorique capable de dépasser l'apparente anomalie de cette réalité. La réponse a été trouvée dans la théorie marshallienne : à l'encontre de l'idée que les économies d'échelle favorisent toujours la grande dimension industrielle, Alfred Marshall avait trouvé dans les économies externes la façon par laquelle les petites entreprises, regroupées sur un même territoire et appartenant au même secteur de production (c'est-à-dire étant étroitement interdépendantes), pouvaient faire aussi bien que la grande entreprise. Rappelons qu'Alfred Marshall écrivait cela à une époque où on assistait déjà à une forte poussée de la concentration industrielle (Rullani, 1984).

Il semble toutefois que Marshall ne distinguait pas, comme il est d'usage maintenant parmi les économistes régionaux, les économies externes d'agglomération de celles de localisation. Cette distinction est pourtant théoriquement importante. En effet, les économies externes d'agglomération génèrent des avantages économiques que les entreprises peuvent tirer des interdépendances territoriales les liant entre elles. Par ce fait, elles ne peuvent être traitées que de façon globale ou holistique. Les économies externes de localisation apparaissent au contraire comme une situation de factum en un point déterminé du territoire. Elles peuvent donc être traitées de façon analytique. Ce n'est toutefois pas un hasard si les premières sont délibérément oubliées au profit d'une réélaboration néo-classique du concept, malgré tous les efforts contraires(Berry, Conkling et Ray, 1976). Le corpus théorique sous-jacent aux économies d'agglomération relève de l'agrégation de comportements atomistes et analytiques individuels, alors que les économies de localisation s'appuient surtout sur les instruments théoriques de l'économie du «bien-être» dans le cadre néo-classique (Smith, 1977) et constituent le pivot de l'interprétation actuelle (légitime) de la problématique écologique (Bresso, 1982).

Il faut dire cependant que Marshall avait en tête les économies externes d'agglomération relevant de la typologie suivante : les économies dérivant de la division du travail entre les entreprises, celles reliées au problème de l'échange d'informations, celles se référant à la formation et donc à l'accumulation de compétence et enfin, celles connexes aux procédés innovateurs (Bellandi, 1987).

La discussion de ces différentes économies allongerait trop cet article. Je me limiterai à rappeler que Marshall ne pouvait pas aller au-delà d'une faible généralisation inductive du modèle du district industriel. D'ailleurs, il ne possédait pas les instruments théoriques adéquats, limités en quelque sorte par la conception classique du développement et la vision néo-classique de l'équilibre optimal (Schumpeter, 1960). 
Plus loin, il sera possible de préciser graduellement divers aspects théoriques, plutôt que des paradigmes structurés à cause des limites mêmes de la théorie, aspects que je ne pourrai pas toujours incorporer sauf sous forme d'insertions ad-hoc dans l'argumentation. Une première possibilité sérieuse de généralisation peut être trouvée dans la théorie transactionnelle de l'entreprise. Mais avant d'y recourir, il faut revenir un peu en arrière.

La question fondamentale sur laquelle se sont penchés les économistes néoclassiques n'était pas sans fondement. Elle avait pour objet d'explorer théoriquement les conditions de l'agir rationnel à l'intérieur d'un système économique. Avec la révolution industrielle ces conditions se sont différenciées du système politique en s'organisant autour de l'argent; et cela, dans un contexte de marché basé institutionnellement sur le droit privé et contractuel bourgeois. L'argent est cet instrument de communication qui, n'ayant aucun contenu en soi, sert à représenter la valeur des ressources à travers les prix que celles-ci acquièrent dans l'échange de marchandises ou, les coûts dans le processus de production.

Cette monétisation (donc cette quantification) généralisée, ajoutée à l'idée d'accumulation et d'aliénation de l'argent, prétend objectiver le contexte économique en transformant la connaissance de celui-ci en un savoir technique ou encore en un savoir techno-scientifique dans la sphère productive, de manière à servir de moyen dans la poursuite des fins (maximisation de l'utilité et du profit) dans le même contexte. La théorie néo-classique a donc cherché à radicaliser le modèle d'action instrumentale dans un contexte économique en faisant abstraction des composantes structurelles de base.

Ce modèle est normatif parce qu'il comporte des prescriptions pour des actions rationnelles et empiriques basées sur l'idéalisation (le «type idéal» selon Max Weber) du modèle d'action implicite dans le processus d'autonomisation du système économique de marché à travers l'argent. De là, la tentative légitime de retrouver un ordre général de la société fondé sur l'interaction des sujets agissant de façon orchestrée et rationnellement par rapport au but économique recherché. Cette tentative ne peut qu'échouer, et cela pour deux raisons. Premièrement, l'argent prend alors une position transcendente par rapport aux ressources tangibles qu'elle sert à valoriser. Elle se différencie de l'argent comme ressource productive et ne se situe donc pas au même niveau logique de production. Deuxièmement, à cause de l'assimilation de l'agir instrumental à l'agir stratégique; alors que le second se différencie du premier quant à l'influence (supposée ajoutée) sur le comportement des autres sujets avec lesquels on entre en interaction. Dans ce contexte, l'action ne devient plus objectivement calculable comme application du savoir technico-scientifique dans un univers externe; elle doit supposer une anticipation, de la part de l'Ego, des réponses de sa contrepartie (l'Alter), comme son anticipation des mouvements de l'Ego. Celui qui agit stratégiquement doit prendre une attitude réflexive qui implique chez l'Ego la présomption d'une triple attitude de l'Ego, de l'Alter et de l'observation externe (Habermas, 1985 : 156-161). 


\section{Le paradigme transactionnel}

Le paradigme transactionnel est justement la revalorisation de la dimension stratégique de l'action. Il permet la construction d'un corpus théorique qui, sans renier radicalement les conceptions théoriques néo-classiques de base, consent à les affranchir des contradictions dans lesquelles elles étaient tombées. Parmi les modèles de l'agir stratégique centrés sur l'interaction entre les sujets et sur la coordination des actions, il y a celui de l'analyse des coûts de transaction (Antonelli, 1984; Mariti, 1980). Cette analyse reprend la thématique des incertitudes dans les échanges en examinant les carences spécifiques du marché en tant que mécanisme de coordination des actions stratégiques dans le transfert des informations sur les valeurs d'utilité des biens à travers la valeur d'échange, de manière à garantir la satisfaction; cette satisfaction de l'abstrait et du présupposé universel soutient, avec le droit à la propriété, le fonctionnement du marché.

Ces carences sont dues à trois causes qui rendent non équivalentes prestation et contre-prestation : a) d'abord les comportements opportunistes, lorsqu'une des contreparties essaie de trouver ses avantages à travers la manipulation de l'information ou suite à une asymétrie dans le pouvoir contractuel; b) puis l' incertitude, c'està-dire, la rigidité des conditions contractuelles par rapport à la flexibilité des rapports nécessaires en relation avec l'évolution des marchés et des technologies; c) enfin l'ambiguïté, c'est-à-dire, la difficulté à évaluer et à interpréter complètement les contenus du savoir-faire et les complications contractuelles possibles des transactions, surtout lorsqu'elles sont particulièrement complexes.

Ce paradigme institutionnaliste spécifie les formes organisatrices qui, malgré le marché, peuvent garantir la réciprocité entre prestation et contre-prestation : a) la hiérarchie, c'est-à-dire les institutions et les grandes organisations publiques (les monopoles d'État, les régies de contrôle de la concurrence) ou privées (les grands groupes industriels) qui définissent explicitement les règles formelles de comportement selon les diverses situations d'interaction et d'interéchange (par exemple, la législation pour la défense des consommateurs, les règles économiques d'échanges entre les filiales industrielles, etc.); b) la communauté, c'est-à-dire les systèmes locaux ou sectoriels d'interactions informelles ou fondées sur l'habitude; ces systèmes ont comme base de comportements un code implicite qui s'est développé graduellement suite à la socialisation des règles de jeu économico-mercantiles spécifiques. Le district industriel serait un mécanisme coopératif qui réunit des aspects de la communauté avec ceux du marché, en limitant les insuffisances de ce dernier.

Arrêtons-nous d'abord à l'aspect coopération avant de discuter de la contribution de la communauté à résoudre les problèmes de mauvais fonctionnement du marché. Dans le district, les sujets économiques sont liés en quelque sorte par un certain rapport amour/haine ou une dialectique concurrence/coopération qui présente des avantages remarquables pour la croissance régionale. La concurrence, en fait, est un mécanisme qui pousse les agents à rivaliser entre eux pour obtenir des 
résultats d'une efficience et d'une efficacité toujours plus grandes. Par contre, la coopération veut réduire les risques de ceux qui entreprendront une activité économique ou qui décideront d'investir dans des produits ou des procédés nouveaux; elle entraîne la coordination des activités complémentaires qui doivent satisfaire des exigences spécifiques de qualités et de quantités. La coopération vise aussi à abaisser les coûts de production en permettant aux entreprises de jouir des économies d'échelle, de la coordination et de l'expérience du district industriel ainsi que de la spécialisation des entreprises tant industrielles que de service.

En ce qui concerne les comportements opportunistes, le district permet à la communauté de faciliter la récolte d'informations contractuelles de contreparties sur les marchés, sur les technologies et sur les nouveaux produits. L'adoption de règles implicites de comportement, par conséquent, les impacts négatifs possibles des décisions sur les marchés et sur les technologies. L'accès à un plus large éventail d'informations (que celles relevant de la contrepartie contractuelle) permet enfin d'évaluer plus globalement les conséquences possibles des transactions, anticipant ainsi d'éventuels problèmes suite, par exemple, à l'adoption d'une nouvelle technologie ou à la gestion du rapport contractuel, problèmes qui autrement n'auraient pas été pris en considération.

Pour poursuivre cette critique de la théorie transactionnelle, il faut maintenant mettre en évidence les limites des présupposés épistémologiques de la théorie néo-classique : le rationalisme et le réductionnisme.

Par ses origines, le rationalisme néo-classique suppose, du côté des agents sociaux, la connaissance parfaite, objective et essentielle. Ce qui nous intéresse ici, c'est la dimension immédiate de la rationalité, c'est-à-dire l'hypothèse d'une connaissance instantanée et non incrémentale de la réalité. Cette présupposition a permis la construction de la théorie néo-classique de l'équilibre général «instantané» où le changement ne peut être introduit qu'en termes de statique comparée; cette statique comparée renonce à vraiment connaître les processus qui ont permis le changement d'un état à un autre. La dimension de l'apprentissage, qui rend irréversible le temps et donc la séquence de choix, échappe irrémédiablement à cette théorie néo-classique parce que, dans des conditions d'apprentissage, il est impossible d'ignorer ce qui s'est passé entre-temps.

De façon idéale, le choix entre les diverses possibilités limitées par les institutions (en l'absence d'hypothèses ad-hoc de généralisations inductives permettant de préserver la théorie) se présente dans la théorie transactionnelle comme celui qui, dans des conditions statiques, assure les coûts minimum de transaction. Ainsi, par exemple, le choix entre l'institutionnalisation et le marché devrait relever du calcul qui permet le rapport coûts/risques le plus bas. Des calculs analogues devraient valoir aussi pour l'alternative marché/communauté.

Ce problème de l'apprentissage nous conduit à la deuxième critique, celle du réductionnisme (l'individualisme méthodologique). On entend par réduction- 
nisme, dans la théorie néo-classique, l'approche méthodologique qui affirme que les faits économiques peuvent être compris seulement par l'étude de leurs composantes élémentaires typiques (par exemple le sujet, agent individuel, rationnel et égoïste) isolables dans leurs relations avec les autres composantes élémentaires. Par agrégation, ces composantes engendrent les propriétés de l'ensemble (ce qui explique la supposée fondation micro-économique de la macro-économie). Malheureusement, comme nous l'avons déjà dit, logiquement il n'est pas possible de faire dériver l'équilibre économique global à partir de telles prémisses.

Si cette critique est, jusqu'à présent, de type logique, on peut aussi faire d'autres réserves fondées empiriquement. Ainsi, plusieurs transactions sur le marché ne se réalisent que dans des «contextes» organisationnels qui supposent «une forme sociale, une histoire globale et un processus d'apprentissage» que toute approche réductionniste ne peut prendre en compte (Biggiero, 1988). La théorie transactionnelle s'appuie fermement sur le présupposé du réductionnisme. Les interactions transactionnelles proviennent de l'interaction entre des sujets agissant rationnellement et individuellement; l'équilibre complexe découle de telles conditions de choix. Les aspects socio-culturels et institutionnels qui constituent les conditions empiriques de choix sont réduits à un univers sans histoire. La théorie transactionnelle ne réussit aucunement à rompre avec ses racines néo-classiques et conserve, par conséquent un statut ambigu.

\section{$4 \quad$ Le paradigme de la complexité}

«L'enracinement régional semble porteur d'une capacité d'évolution localisée à deux niveaux logiquement connexes. Le premier niveau concerne l'ouverture régionale à l'acquisition d'énergie et d'information (...). Le deuxième met en cause le concept de système sui-référentiel (ou «autocréateur») développé récemment dans les prolongements de la théorie des systèmes. La principale hypothèse de ce concept théorique est que les éléments d'un système sont produits et reproduits par ce même système, selon un code de différentiation sui-référentiel. Ce mouvement circulaire de relations reproductives internes est une conséquence logique de l'ouverture du système à son environnement, puisque en l'absence de celui-ci, le système cesserait de vivre par lui-même, c'est-à-dire comme différent de son environnement» (Tinacci Mossello, 1987 : 115). Mais il faut expliciter ces concepts qui, ainsi formulés, peuvent être vides de sens.

Pour cela, j'aurai recours aux formulations de Niklas Luhmann qui en a radicalisé l'application en sociologie en partant des premières élaborations du circuit de la métabiologie de la connaissance. Luhmann (1989) part d'une définition commune de la complexité d'un système et de son environnement en énonçant les postulats suivants : 
a) l'environnement d'un système est toujours plus complexe que le système luimême; aucun système ne peut associer sa propre performance aux éléments et aux relations avec son environnement;

b) pour faire face à la complexité de l'environnement, le système doit le réduire en choisissant entre plusieurs expériences possibles; mais en agissant ainsi, il conserve la complexité comme un objectif non souhaité (Luhmann, 1973);

c) différencier le système de son environnement est une condition indispensable pour réduire la complexité; et la compréhension de celui-ci doit se fonder sur un code de communication interne au système;

d) en agissant ainsi, le système transforme les données provenant de l'environnement en informations en séparant ces dernières car autrement, elles ne pourraient être connues du système et ne pourraient lui servir;

e) cette sélection face à la complexité de l'environnement et aux sens des informations transmises est fondée sur un code interne au système; le système est ainsi sui-référentiel en ce sens qu'il traduit l'environnement externe à partir de sa propre organisation informationnelle, (le système demeure refermé sur luimême dans son ouverture à l'environnement);

f) si dans cette communication sui-référentielle, les éléments du système sont insuffisants pour réduire la complexité de l'environnement de manière souhaitée, le système peut se différencier en générant des sous-systèmes spécialisés en élaborations spécifiques du sens des informations, par un processus d'autocréation;

g) cette génération interne de sous-systèmes, qui fait en sorte que le système devienne leur environnement, est un processus de différentiation fonctionnelle;

h) cette diversité essentielle est le niveau de différentiation du système nécessaire pour faire face et réduire autant que possible la diversité de l'environnement sans que le système en soit menacé d'extinction.

L'idée que Luhmann a des systèmes sociaux est très abstraite car il ignore la dimension matérielle des connextions intersystémiques. Dans l'optique du système social complexe, la seule interaction matérielle significative survient avec l'environnement écologique. Mais cette interaction représente pour le système une valeur seulement en autant qu'elle est médiatisée par le code de communication, qui est l'argent, du sous-système économique, interne au système social; l'interaction matérielle n'a pas de valeur en soi, pas plus que cette valeur n'est déterminée par l'environnement. Si nous devons, par contre, rendre opérationnelle la définition du système complexe pour des fins d'économie régionale, nous devons garder à l'esprit que les interactions de la région avec son environnement s'effectuent entre soussystèmes régionaux, c'est-à-dire avec d'autres sous-systèmes qui partagent (du moins, en partie) les mêmes codes de communication. Elles font donc que le système n'est plus fermé de façon sui-référentielle, mais devient plus ouvert. La diversité de 
l'environnement devient hétéro-définie et ne peut plus être réduite à une volonté du système. Nous devons donc nous tourner vers une définition plus large de la complexité.

Angelo Turco (1988) définit la complexité d'un système en fonction du nombre de ses éléments, du grand nombre de relations possibles entre ces éléments, entre ceux-ci et l'environnement, et, enfin, du nombre de finalités du système même. Le système peut être menacé dans son existence par la diversité et par la variabilité de l'environnement; un excès de diversité et de variabilité le place devant des tensions évolutives pour lesquelles il pourrait ne pas disposer d'éléments et de soussystèmes suffisamment spécialisés pour les affronter.

L'autonomie du système, et donc sa capacité de répondre aux tensions dues aux pressions de son environnement pour qu'il évolue, dépend : (1) du caractère aléatoire de ses relations internes; plus grand est le nombre de possibilités en tous sens des relations existantes entre les éléments (bi-directionnel versus monodirectionnel), plus grande est la capacité du système à répondre de façon flexible, c'est-à-dire de façon adéquate à l'aide d'un processus interne capable d'affronter toute perturbation; (2) de la maximisation des conditions aléatoires, c'est-à-dire de l'intensification du nombre de relations relevant du hasard; (3) de la stabilisation de ces conditions aléatoires, c'est-à-dire de la capacité du système à contrôler toute relation supposant un affrontement avec l'environnement. Chaque enracinement du système en termes de croissance de la complexité signifie une hausse de la potentialité d'autoconservation et de développement du système lui-même.

La conception systémique de la réalité est de ce point de vue dynamique, selon un processus «d'autocréation». Celui-ci se réalise à travers l'action de chaque membre particulier ou sujet du système; celui-ci peut trouver à l'intérieur du système les conditions pour sa propre auto-organisation. Un système complexe est, en fait, décentré puisque chacun de ses éléments est un centre d'évolution autonome, lié au système par des rapports nécessairement hiérarchiques et surtout par des réseaux de relations. Le pouvoir de ces éléments par rapport au système se définit «comme la capacité d'un sujet à exercer sa propre autonomie dans des champs interactifs marqués par le hasard et l'incertitude dans le choix entre diverses possibilités» (Turco, 88: p. 50). Les éléments du système sont à leur tour des systèmes dotés d'une complexité spécifique, si bien que le système est différent de la somme de ses composantes. Il est en même temps plus et moins. La globalité n'est pas la somme de ses sous-systèmes et simultanément, le local n'est pas partie du global; on ne peut pas connaître le local en partant seulement du global, pas plus qu'on ne peut définir ce dernier comme un agrégat de parties ou de comportements identiques. C'est pour cette raison que dans l'optique du système, les éléments sont à la fois l'environnement du système lui-même, en correspondance avec le système et son environnement comme l'est l'environnement du système avec lequel les éléments sont en relation. Puisque chaque élément ou sujet décisionnel est autonome à l'intérieur du système, l'auto-observation du système se fait par l'analyse de la signification de chaque élé- 
ment ou des sous-systèmes du système. En partant de l'analyse des tensions du système, chaque élément est en mesure de favoriser, en agissant sur la marge d'autonomie, le développement de nouvelles stratégies d'action pour générer la croissance du système même, et ainsi sa transformation et sa différention fonctionnelle.

Le district industriel relève de cette théorie métabiologique de la complexité. Le district peut se définir à plusieurs niveaux, soit (1) le système lui-même; (2) le sous-système économique des entreprises avec les sous-systèmes politiques (les villes ou communes) et le sous-système communautaire; (3) enfin, les éléments (les entreprises et les agents privés). Il ne peut donc qu'être très riche en relations internes. Ces relations sont aléatoires, car elles sont nombreuses et ont une forte composante informelle (ni hiérarchique ni mono-directionnelle). Le processus d'autocréation est assuré par la capacité de générer une différentiation fonctionnelle quand ses entreprises ou ses agents entrevoient la possibilité d'affaires nouvelles avec d'autres éléments du district ou avec son environnement. Ce processus d'autocréation est, en outre, possible quand celui qui désire s'impliquer cherche localement les ressources nécessaires pour soutenir son activité et se nourrit éventuellement de la disparition (faillites...) d'autres entreprises qui n'ont pu faire face aux tensions provenant de l'environnement (par exemple, les nouvelles technologies) et qui ont ainsi libéré des ressources (la négentropie peut provenir aussi de la libéralisation de l'entropie). Le district peut être cependant pauvre en relations entre les éléments et l'environnement s'il relève d'une culture mono-industrielle, s'adresseà un seul marché (donc, pas très différencié) et utilise des intrants technologiques très sélectionnés. Ainsi, une évolution rapide des technologies ou des marchés pourrait le perturber de façon importante.

Les présupposés épistémologiques d'une telle réinterprétation sont, pour la majeure partie, à l'opposé de ceux des néo-classiques (Biggiero, 1988). L'appréhension ou la connaissance du district est subjective lorsqu'elle est formulée dans le langage de l'observateur (ceci vaut soit pour la connaissance imputable aux sujets observés, soit à celle obtenue par l'observation scientifique, parce qu'elle n'est pas autre chose que la tentative intuitive d'un sous-système social qui objective d'un point de vue subjectif ce même système social). Cette appréhension ne peut atteindre le savoir final et parfait. La rationalité ne peut être ni parfaite, ni absolument limitée; elle est relative au code linguistique spécifique du sous-système, produit historique d'un procédé d'apprentissage dans l'interaction entre le système (le district) et son environnement. La vision du monde est holiste et se satisfait évidemment du besoin de représentation, par exemple deséconomies externes d'agglomération. Le système (district) évolue selon un processus irréversible et selon diverses réponses contingentes aux tensions de l'environnement. Il s'explique ainsi aujourd'hui; mais il pourrait être complètement différent si la succession des réponses contingentes et des processus connexes de l'apprentissage avaient suivi un cours différent. Le système (district) n'est qu' un produit contingent historique. Il s'en suit que la vision du monde n'est pas déterminée. L'évolution se fait par des variations qualitatives. 
En acceptant une vision systémique du monde, je ne voudrais pas me laisser aller à un irrationalisme et à un subjectivisme post-moderne doté de complexité. Je reprendrai donc quelques observations relatives à la rationalité communicationnelle. Elles serviront en fait à intégrer le modèle que je proposerai plus loin.

\section{Le paradigme de la communication}

Dans un passage particulièrement éclairant, Giocomo Becattini affirme : «Si nous pouvons classer les phénomènes sociaux par catégories purement cognitives, ils seront cohérents, comme cela doit être naturellement, de façon à rendre plus facile et exacte l'obtention de résultats d'un cas à l'autre à l'intérieur de la même classe. Si, cependant, le but de la recherche est de fournir, directement ou indirectement, des critères pour l'action, alors la classification adoptée devra tenir compte de la manière dont ils représenteront la réalité en question. Cela revient à dire que le chercheur réfléchissant sur la réalité sociale ne peut superposer purement et simplement sa classification scientifique avec celles qui, pour des raisons tirées de l'histoire, prévalent chez les «agents»; à moins que ceux-ci ne soient des fonctionnaires publics chargés de réaliser le plan ou bien de simples citoyens subissant ce plan. Après avoir étudié la réalité sociale avec sa propre méthode, il doit la représenter selon la même réalité (si on peut ainsi dire) que s'en font les agents, et surtout bâtir des ponts permettant à ceux-ci de retrouver leurs propres termes. S'il ne réussit pas, chaque conclusion définie en ses termes et chaque politique suggérée produira le plus souvent des résultats inattendus et peut-être non désirés» (Becattini, 1987 : 31-32).

Or, l'idée que l'observateur scientifique fasse partie intégrante du système qu'il observe en tant que sous-système de celui-ci, est aussi présente dans la théorie systémique; et cela est présenté comme étant une grande avancée de la théorie de la connaissance (!). Mais cette idée d'un système s'observant lui-même acquérant la connaissance du monde social observé et agissant en communication avec lui par l'entremise de son propre code linguistique spécifique, suppose un rapport externe entre l'observateur et l'observé. Par contre, ce que Becattinia en tête est probablement un rapport interne entre l'observateur et l'observé; cette connexion passe par l'intersubjectivité de la communication tirée de la communauté linguistique à laquelle l'observateur appartient; cette inter-subjectivité est une précondition nécessaire afin que soient intégrés les plans d'action pour les deux parties. Cette idée a une histoire très longue et trouve ses racines en linguistique, en phénoménologie et en herméneutique.

Une telle négociation systématique, lucide et incisive, entre ces deux parties peut être expliquée à l'aide d'une proposition de Habermas (1970) faite il y a une vingtaine d'années. L'idée de base est que les sciences sociales se retrouvent face à une double exigence interprétative : d'un côté, il y a le système linguistique scientifique à l'intérieur duquel sont formulées les propositions et qui définit les limites des expériences possibles du chercheur; de l'autre côté, la réalité sociale 
apparaît comme une totalité pré-interprétée par les sujets qui la constituent; et il n'est possible de la connaître qu'en participant à l'interaction communicationnelle de façon à comprendre les significations qui expliquent la vie des agents (Vagaggini 1982 : 330-334). C'est le problème de la double herméneutique.

Cette relation cognitive interne sert à l'analyse systémique pour laquelle il n'y a que des agents «désubjectivisés» en systèmes sui-référentiels qui tentent d'isoler le monde tout en y étant reliés exclusivement par un code de communication. Ce moyen n'est possible que si les messages communiqués ne supposent pas une appartenance intersuggestive de sens, de significations et de validité.

C'est pour cela que j'ai introduit provisoirement le concept de système culturel ou n'importe quel autre système équivalent en parlant du district industriel. Il faut supposer pour celui-ci l'existence d'un code de communication qui fonctionnerait comme médium externe entre les agents et qui permettrait de définir les limites du système culturel en tant que sous-système sui-référentiel constitué comme différentiation fonctionnelle du système «district industriel». Mais je ne vois pas comment cela pourrait se faire car la culture est organisée autour du langage ordinaire et fait fonction de moyen de communication du système social. Mais contrairement à la monnaie, cet instrument de communication est étroitement lié à la vie des participants de la communauté (de communication) au point de ne pas pouvoir fonctionner comme moyen spécialisé externe aux sous-systèmes individuels : sa position logique est semi-transcendantale.

L'approche systémique, par conséquent, a un problème analogue à celui du paradigme transactionnel. Lorsque ce dernier renvoie la communauté à ses fondements historiques, l'approche systémique présente indubitablement un modèle qui peut fonctionner pour la dimension économique ou pour la société dans sa totalité, en réduisant la culture à un sous-système spécialisé. La communauté, en ce qu'elle semble représenter une composante-clé pour l'interprétation des conditions de succès du district (dans tous les paradigmes jusqu'ici étudiés et pour utiliser encore ici une métaphore du langage systémique), reste reliée à l'environnement du système des entreprises; mais cet environnement demeure en définitive inexploré dans ses composantes fondamentales.

Je reprendrai donc les plus récentes propositions de J. Habermas (1979, 1985, 1986, 1987) dans sa «théorie de l'agir communicationnel» (sans oublier les contributions de K.O. Apel, 1989). Habermas base explicitement une partie de ses considérations sur une ré-élaboration tant ontogénétique que philogénétique et comprenant des composantes cognitives morales et individuelles; ces propositions relèvent aussi des différentes prospectives d'action et de l'épistémologie génétique de Piaget (Piaget et Garcia, 1985). Habermas part de l'hypothèse de l'émergence des composantes structurelles de la rationalité occidentale comme procédé évolutif non contingent, entendu comme le fait qu'il existe dans l'histoire de la société une logique de développement non déterministe. Cette logique peut, en effet, se concrétiser en actions, connaissances, institutions et individualités sous la forme d'implantation 
d'un potentiel de savoir disponible et ontogénétique; elle peut s'actualiser dans des conditions empiriques de crises du modèle d'intégration, de régularisation et de contrôle d'une société donnée. Ces conditions se présentent donc comme des affrontements évolutifs : la transition à un stade plus élevé de rationalité constituerait un processus improbable d'apprentissage, d'abstraction et d'universalisation des conceptions sociales. Ceci serait coupé des perspectives concrètes ou relèverait de systèmes d'autorité ou d'images abstraites du monde dogmatiquement définies. Les perspectives d'action peuvent historiquement être différenciées d'un côté entre l'agir instrumental et stratégique et l'agir communicationnel, pour celui qui anticipe une volonté d'entente par l'interaction (c'est-à-dire une connaissance inter-subjective prétendument valide). L'agir communicationnel se présente comme une forme d'action par laquelle il est possible de soumettre à l'argumentation et ainsi d'entraîner un assentiment pour agir ou non; cependant, cette argumentation doit être organisée en propositions assertives, régulatives ou expressives; elle relève de la vérité, de la justesse et de la véracité si elle touche (1) à des connaissances sur l'univers objectif (les faits du monde naturel et social), (2) à des relations interpersonnelles avec le monde social (les rôles et normes légitimes) et (3) à des auto-représentations de l'univers subjectif (les besoins, les désirs et les émotions).

Le monde vivant est complémentaire à l'agir communicationnel (Schutz et Luckmann, 1974). Par cette affirmation, Habermas veut dire que l'appartenance aux connaissances, aux valeurs, aux normes et aux modèles de comportements peut être reconnue inter-subjectivement comme valide à l'intérieur d'une société. Les composantes structurelles de cette appartenance, elles-mêmes différenciées de la modernité, sont la culture ou l'ensemble des images du monde et des valeurs partagées, la société, ou l'ensemble d'organisations légitimes et de valeurs institutionnalisées, et enfin la personnalité, ou l'ensemble des compétences qui rendent un individu capable de parler et d'agir. Les images du monde sont devenues tellement dépendantes des concepts abstraits de ce même monde, en termes de contenus cognitifs concrets, d'organisations d'origines juridiques et de morale basée sur de principes universels plutôt que sur des particularités éthiques que les capacités d'action et de connaissance ne peuvent plus produire une identité distincte d'orientations culturelles traditionnelles et des encadrements pour des rôles concrets.

Le monde vivant constitue donc une réserve pour l'agir de communication. Parce que c'est seulement à partir de l'appartenance au savoir et à la linguistique que peuvent devenir problématiques et relever de l'argumentation divers aspects particuliers du monde objectif, social et subjectif; mais ce monde se nourrit en même temps de nouvelles images du monde, de normes légitimes et d'agents formés par la communication argumentative. C'est dans la dimension de l'agir communicationnel que se réalisent (1) la reproduction cultur elle qui assure dans la diversité la continuité de la tradition et une cohérence du savoir suffisant pour orienter la pratique, (2) l'intégration sociale des membres en soutenant l'acquisition des capacités d'agir généralisées et en adaptant à l'histoire les vies individuelles et collectives (Habermas, 1987 : 343). 
La surcharge de communication permet la rencontre d'un monde vivant réalisé, où chaque image du monde, chaque norme juridique ou morale requièrent une prise de position des membres pour l'interaction au lieu de l'intériorisation et de la continuité des traditions culturelles et éthiques concrètes. Cette surcharge rend nécessaire la production de communications abstraites et spécialisées qui réduisent les circuits permettant une reconnaissance inter-subjective explicite pouvant être validée. L'instrument monétaire et le pouvoir remplissent ce rôle à l'intérieur des sphères économiques et politico-administratives de la société. Ces moyens permettent que les participants à l'interaction sociale y assument une attitude non plus interne mais externe à elle. Dans le monde vivant, l'institutionnalisation du droit à la propriété et à la vie privée transforme le marché en un système contrôlé par le moyen de communication qui représente l'argent, tandis que l'institutionnalisation des organisations rendues légitimes grâce aux procédures formelles et abstraites de normalisation structure l'organisation hiérarchique comme un système contrôlé par le moyen de communication du pouvoir qui, moyennant des décisions obligatoires, poursuit des objectifs sociaux bénéfiques pour tous.

À son tour, le monde vivant alimente les systèmes économiques et politicoadministratifs de connaissances organisées et pouvant être communiquées (comme le suggère Rullani) sous la forme abstraite d'un savoir technico-scientifique. Cela signifie par contre que n'importe quelle connaissance objective du contexte social (comme savoir organisé ou du marché, etc.) crée des légitimations et des motivations de l'action conformes aux exigences de régulation et de contrôle des deux soussystèmes.

Les idées de Chomsky $(1981 ; 1984)$ sur l'agir communicationnel me permettent ici de répondre à l'exigence de la gouverne d'une compétence linguistique grammaticale, et donc syntaxique, qui est une condition nécessaire afin que l'interaction linguistique puisse avoir lieu.

De la même manière, je vois dans la dimension du travail la composante syntaxique de base autour de laquelle sont organisés l'agir instrumental et stratégique ainsi que la reproduction matérielle de la société. D'un autre côté, d'un point de vue ontogénétique, le travail se trouve aussi dans la manipulation des objets provenant du développement de la compétence cognitive de l'enfant. Il en est de même dans la dimension du langage qui provient de la maturation, de la compétence communicative et du processus de socialisation et de la mise en perspectives du rôle de l'enfance. Analogiquement, sur le plan philogénétique, nous pouvons assumer que c'est seulement dans les structures du travail et du langage que se sont accumulés les développements sur la reproduction de la vie spécifique humaine en tant que condition humaine et partie de l'évolution sociale. Le travail et le langage sont antérieurs à l' homme et à la société (Habermas, 1979 : 112).

Cette théorie de l'agir communicationnel de Habermas apparaît extrêmement puissante. Elle permet la différentiation nécessaire des niveaux de discours en termes de structures profondes ou superficielles d'universalisme et de particularisme 
de la dimension pragmatique, et de projection interne et externe de l'action, au point de favoriser (en utilisant une métaphore systémique) la variété fonctionnelle nécessaire pour affronter la complexité de l'environnement.

Mais laissons maintenant ces précisions théoriques et regardons comment elles s'appliquent au district industriel.

a) L'approche transactionnelle présente en soi un problème logique à différents niveaux de discours. Ainsi, communauté, marché et hiérarchie ne sont pas interchangeables au même niveau. La dimension communautaire appartient à la prospective du monde vivant. Elle peut être comprise à travers des modèles d'interaction fonctionnant dans le district ou dans n'importe quel autre système d'entreprises, dont le marché et la hiérarchie comme réserve de motivation et de connaissance sont les deux dimensions. En conséquence, la communauté n'est pas une alternative ni au marché ni à la hiérarchie, mais plutôt la condition d' existence de l'un ou de l'autre. C'est pour cette raison que l'approche transactionnelle ne réussit pas à comprendre la dimension communautaire. Elle réduit à la base l'agir rationnel par rapport au but, justement parce qu'elle est organisée autour d'une orientation différente de l'action.

b) Un problème analogue limite l'approche systémique. La communauté n'est pas un système organisé autour d'un mécanisme abstrait qui a besoin, pour pouvoir fonctionner, d'une légitimisation externe. Ici le moyen de communication est le langage naturel, et on ne peut parler d'un fondement institutionnel. Le langage naturel fonctionne à l'intérieur des formes de vie communautaires, et sert à les reproduire par l'entremise de l'agir communicationnel.

c) Cela ne signifie pas, cependant, que l'approche néo-classique du marché et celle systémique de la complexité soient dépourvues de fondement. Mais ces approches ne permettent qu'une explication partielle à l'intérieur d'une discussion plus générale sur le district. L'approche néo-classique permet de prendre conscience des dimensions pragmatiques universelles d'un modèle d'action rationnelle à l'intérieur d'un système institutionnellement organisé autour du marché. Cet aspect de l'action rationnelle nécessaire échappe à la critique systémique. D'un autre côté, l'approche systémique développe le processus de différentiation fonctionnelle du système «district industriel» comme réponse holiste d'un organisme à son environnement économique hyper-complexe, disposant d'une grande diversité et d'une variabilité qui le menace d'extinction.

d) Ne pas différencier suffisamment les niveaux du discours peut conduire à combiner le paradigme des économies externes marshallienne au paradigme systémique. En agissant ainsi, seule l'analyse marshallienne explique le district industriel, en replaçant chaque autre forme de district dans le concept plus vaste de système territorial d'entreprises. Ceci ne permet pas de se rendre compte que les conditions soutenant les relations inter-entreprises d'un district sont profondément différentes de celles d'un pôle de développement et aussi de celles d'un parc 
technologique. En conséquence, on doit adopter une attitude exteme, objectivante, par rapport au district défini par un ensemble de conditions socio-culturelles et économico-productives à satisfaire.

Du point de vue socio-culturel, le système d'entreprises ne doit pas être dominé par les classes sociales supérieures et par leurs investissements (les grandes entreprises). La part du tertiaire doit être limitée, le nombre de femmes qui y travaillent doit être élevé et l'entrepreneuriat doit être répandu et caractérisé par la figure sociale du petit entrepreneur industriel (en distinguant bien l'industrie de l'agriculture). Cette perspective tient donc compte d'un système de variables orienté vers l'action liée à une approche cognitive, éthique et socialisante conventionnelle. Cette approche est fondée sur la tradition; c'est-à-dire qu'elle est justifiée par le fait que la force de travail joue dans des espaces de la conscience universelle de classes. Quantà l'aspect de l'éthique conventionnelle, cela signifie une attitude de conformité aux attentes personnelles de comportement quant au rôle social souhaité, aux images stéréotypées du comportement «naturel» de la majorité et à l'ordre social; ce comportement doit reproduire tout cela, en visant à maintenir activement, à soutenir, et à justifier un tel ordre, de façon à s'identifier comme des membres d'un groupe cohérent (Habermas, $1979: 60$ ).

La dimension communautaire traditionnelle se caractérise par la différentiation systémique entre une économie de marché autogéré et une modernité en tant que processus idéal qui se transforme progressivement. La conscience bourgeoise se prétend orientée vers l'universalisme plutôt que vers le particularisme et est en contradiction avec de tels fondements éthiques et cognitifs.

Si nous devons extrapoler dans le temps les tendances du district, il faut prévoir un écart croissant entre ce monde communautaire et les structures qui le constituent. Ceci s'explique par l'ouverture à une compétition plus ouverte, une confrontation avec un contex te économique plus large et surtout l'entrée des modèles informatiques de communication technicalisées (Rullani, 1987). Ce changement entraînera des actions de plus en plus différenciées jusqu'à remettre en question l'agir de communication. Il faut alors s'attendre à une révolution consciente des composantes rationnelles des images du monde et des connaissances technico-scientifiques utilisées et de l'enracinement institutionnel des modèles d'interaction. On verra apparaître des formes de coopération plus explicites et moins enracinées dans les composantes traditionnelles de l'éthique locale. Ces formes de coopération soutiendront la socialisation et produiront des structures professionnelles construites autour d'identités individuelles cosmopolites. Alors, ces structures permettront une disponibilité généralisée pour assumer les rôles de travail intégrant les autres aspects de la vie quotidienne.

e) La croyance selon laquelle l'enracinement du district industriel dans la communauté locale et traditionnelle pourrait persister avec succès en maintenant le système dans un état stationnaire se base sur le fait que, dans les grandes entreprises, une profonde transformation agit et réévalue la composante socio-culturelle et 
motivationnelle du personnel. Les grandes entreprises se sont restructurées dans les dernières années en se divisant fonctionnellement en sous-systèmes stratégiques autonomes, avec des objectifs de marché, de technologie ou de production différenciés, afin de faire face à un environnement économique évoluant vers la diversité et la variabilité. Une telle différentiation fonctionnelle signifie que l'entreprise dans son ensemble maintient le contrôle sur les sous-systèmes, d'une part grâce à l'argent et au pouvoir sous forme de techniques de planification et de contrôle de gestion et, d'autre part, à l'aide du système normatif de l'entreprise. Mais pour cela, elle doit en même temps s'assurer d'un double enracinement de ses membres à l'entreprise. Elle est ainsi en mesure de reproduire les images de communauté et d'unité de son univers. Mais cela suppose aussi de la part des membres une conscience qui va au-delà des conventions et de l'évolution de la structure professionnelle; cette évolution provient des nouvelles exigences de contrôle et de l'informatique appliquée aux procédés de l'entreprise, requérant un niveau de formation supérieur des employés.

Une scolarisation élevée et une socialisation poussée dans un contexte familial orienté vers de nouvelles valeurs (par exemple d'autonomie), de nouvelles formes de cohésion et d'entente permettent aux jeunes l'accès à des formes d'apprentissage toujours plus abstraites et plus ouvertes. Cet apprentissage, transformant les rôles sociaux concrets et les relations avec l'environnement économique et politicoadministratif, complète la maturation de leur propre conscience morale. Cette conscience atteint un certain niveau dans lequel l'accession à un système normatif donné permet de dépasser pour approcher un niveau dans lequel se fondent de nouvelles normes et règles de comportement nécessaires au nouvel environnement.

En effet, dans une entreprise, contrairement à la société, l'autorité est fondée sur le droit à la propriété des moyens de production. Elle a pour ainsi dire, un fondement axiomatique. Sous une telle forme de gouverne, les ordres proviennent de la hiérarchie et n'ont pas besoin de justification quant à leur légitimité, étant implicitement contenus sous forme dogmatique dans l'acceptation du système d'autorité.

Dans ces conditions de conscience post-conventionnelle et dans les interactions quotidiennes, chaque impératif a besoin de justification. Le destinataire de l'impératif doit prendre position face aux ordres en les acceptant comme valide ou non. Seul un enracinement soutenant l'interaction entre l'entreprise et l'employé, dans un monde rationalisé par les moyens de l'agir de communication pour obtenir une entente, peut permettre de dépasser toute contradiction entre le système d'autorité fondé sur une conscience conventionnelle et la propre conscience morale de l'individu dans la société qui évolue vers un niveau post-conventionnel.

Grâce à ces procédures rationnelles, la participation du personnel au processus de définition des objectifs sectoriels et à la formalisation du budget est le stratagème que l'entreprise peut utiliser pour sortir de l'impasse. Elle peut aussi profiter de l'enracinement ultérieur dans la démarche d'actions rationalisées de la modernité, c'est-à-dire dans l'agir stratégique, en ajoutant divers stimuli économi- 
ques pour que l'employé adhère aux objectifs qu'il a contribué à définir. En même temps, ce n'est plus la position d'autorité, mais le leadership (comme reconnaissance inter-subjective d'une crédibilité et d'un guide moral) et la réputation (comme reconnaissance inter-subjective d'une crédibilité et d'un guide cognitif) qui peuvent réduire la nécessité d'une entente communicationnelle et le questionnement des ordres dans la pratique quotidienne. Leadership et réputation constituent des moyens spécialisés pour l'adhésion consentie. En agissant ainsi, l'entreprise agit en fonction de ses intérêts. Parce qu'elle peut se diviser en pôles décisionnels indépendants et hautement responsabilisés sans perdre le contrôle complexe de la structure même. Un contrôle de la force de travail, non plus axée sur la seule présence des travailleurs mais sur la participation aux objectifs agencés et définis rationnellement, permet pour la première fois dans l'histoire de dépasser le modèle de concentration des travailleurs dans la manufacture, qui fut à la base de la révolution industrielle anglaise. Cette combinaison de la diversité et la variabilité de l'environnement ainsi que la flexibilité offerte par les technologies informatiques est essentiellement socio-culturelle dans le cadre d'une conscience post-conventionnelle. Dans la terminologie marxiste, on peut parler d'une nouvelle cohérence entre forces productives et rapports de production, ce dont ne parle pas Thomas Maldonado (1987) dans sa lucide défense de la modernité. Ce dernier ne dit rien en outre sur le travail décentralisé. Ce travail décentralisé n'est malheureusement qu'un slogan, non seulement commercial mais aussi culturel, de l'industrie informatique. Il est une potentialité non-actualisée niée par les faits et le pouvoir conservateur. Ce pouvoir est le plus souvent incarné par le personnel qui craint de perdre ainsi les instruments formels et traditionnels de contrôle et qui a de la difficulté à s'émanciper d'un demi-siècle de traditions et d'études sociologiques sur l'organisation du travail ouvrier. Hors contexte, ce pouvoir conservateur relève aussi de ce qui reste de l'archéologie intellectuelle marxiste orthodoxe ancrée nostalgiquement dans les vieux dogmes en accord avec les forces sociales les plus conservatrices.

C'est probablement la récupération dans les dernières années des forces de compétitivité par la grande entreprise qui explique une crise montante dans la petite entreprise. Cela s'explique par le fait que la grande entreprise a réussi à se rebâtir en exploitant à fond quelques-unes des potentialités offertes par la rationalisation des orientations de l'action autant dans le contexte de l'agir de communication que dans celui de l'agir stratégique. Ce changement ne s'est pas encore réalisé dans les districts industriels marshalliens, là où l'enracinement de la communauté a des connotations traditionalistes et conventionnelles. Aussi, si nous devions retrouver un état stationnaire, nous devrions le situer comme une évolution à partir des districts nonmarshalliens (de type Biella, Cantû, Vigevano, etc.), dans lesquels ont été dépassées, en partie, les structures conventionnelles de la conscience à base des composantes sociales (le modèle de Sforzi, 1987) représentant l'intermédiaire entre l'intégration sociale et la reproduction culturelle socialisante. Dans ces districts, les parcours de la rationalisation peuvent être différents de ceux suivis par la grande entreprise, par exemple, en exploitant les options non valorisées dans ce contexte. 


\section{BIBLIOGRAPHIE}

Antonelli, C., (1984), Cambiamento tecnologico e impresa multinazionale. Il ruolo delle reti telematiche nelle strategie globali, P. Angeli, Milan.

Apel, K. O, (1989), «Etica del discorso come etica della responsabilità e il problema della razionalità economica», Banca Toscana, Studi e Informazioni, Quaderni 24, Florence.

Becattini, G., (1987), «Introduzione : Il distretto industriale marshalliano : cronaca di un ritrovamento», dans G. Becattini (éd.), Mercato e forze locali : il distretto_industriale, Il mulino, Bologne, pp. 7-34.

Bellandi, M., (1987), «La formulazione originaria», dans G. Becattini, op. cit., p. 35-48.

Berry, B.J., Konkling,E. C. et Ray, D. M., (1976), The Geography of Economic Systems, Prentice-Hall, Englewood Cliffs (N. J.).

Biggiero, L., (1988), «Sull'accettabilità della teoria neoclassica», Economia e politica industriale, no 60, pp. 199-244.

Bresso, M., (1982), Pensiero economico e ambiente, Loescher, Turin.

Chomsky, N., (1981), Riflessioni sul linguaggio, Einaudi, Turin, traduction.

Chomsky, N., (1984), Regole e rappresentazioni, Il Saggiatore, traduction, Milan.

Habermas, J., (1970), Logica delle scienze sociali, Il Mulino, traduction, Bologne.

Habermas, J., (1979), Per la ricostruzione del materialismo storico, Etas Libri, Milan.

Habermas, J., (1985), Etica del discorso, Laterza, Bari.

Habermas, J., (1986), Teoria dell' agire comunicativo, II Mulino, Bologne.

Habermas, J., (1987), Il discorso filosofico della modernità, Laterza, Bari.

Luhmann, N., (1989), Communicazione ecologica. Può la società moderna adattarsi alle minaccie ecologiche?, F. Angeli, Milan.

Luhmann, N., (1973), «Il senso come concetto fondamentale della sociologia», dans J. Habermas et Luhmann, N., Teoria della società o tecnologia sociale. Che cosa offre la ricerca del sistema sociale?, Etas Kompass, Milan, pp. 14-66.

Maldonado, T., (1987), Il futuro della modernità, Feltrinelli, Milan.

Mariti, P., (1984), Sui rapporti tra imprese in una economia industriale moderna, F. Angeli, Milan.

Piaget, J. et Garcia, R., (1985), Psicolgenesi e storia delle scienze, Garzanti, Milan.

Rullani, E., (1984), «Teoria ed evoluzione dell'impresa industriale». dans M. Rispoli, L'impresa industriale, Il Mulino, Bologne, pp. 15-86.

Rullani, E., (1987), «L'impresa come sistema artificiale : linguaggi e apprendimento nell'approccio evolutivo alla complessità», Economia e politica industriale, no 56, p. 215-243.

Schutz, A. et Luckmann, T., (1974), The Structures of the Life-World, Heinemann, Londres.

Sforzi, F., (1987), L'identificazione spaziale, dans G. Becattini, op. cit.s pp. 143-167.

Smith, D. M., (1977), Human Geography : a Welfare Approach, Arnold, Londres.

Tinacci Mosselo, M., (1987), «Economie di agglomerazione e sviluppo economico», dans G. Becattini, op. cit., p. 93-116.

Turco, A., (1988), «Verso una teoria geografica della complessità», photocopies, Milan.

Vagaggini, V., (1982), Le nuove geografie. Logica teorie e metodi della geografia contemporanea, Herodote, Genève-Ivrea. 